\title{
Impacto del PIB sobre los Ingresos Tributarios del Ecuador durante el periodo $2000-2018$
}

URL: http://revistas.uta.edu.ec/erevista/index.php/bcoyu/article/view/716 Dol: http://dx.doi.org/10.31164/bcoyu.22.2019.716

\section{Margarita Jumbo - Benítez ${ }^{1}$}

Fecha de recepción: 19 de mayo de 2019

Fecha de aceptación: 19 de agosto de 2019

\section{Resumen}

La investigación mide el impacto del Producto Interno Bruto del Ecuador, en la recaudación tributaria del Impuesto al Valor Agregado, Impuesto sobre la Renta; y, Derechos Arancelarios frente a las Importaciones, desde el año 2000 hasta el junio del 2018 con una perspectiva para corto y largo plazo. Los resultados de la elasticidad impositiva a largo plazo, se obtuvieron mediante Mínimos Cuadrados Ordinarios, y los de corto plazo con el Modelo de Corrección de Errores. Los parámetros estimados indican que ante un incremento del PIB, la recaudación del IVA e ISR crece, igualmente los Derechos Arancelarios frente a las Importaciones.

Palabras clave: Impuestos, elasticidades, cointegración.

\section{Abstract}

The research measures the impact of the Gross Domestic Product of Ecuador on the tax collection of the Value Added Tax, Income Tax and Tariff Rights against imports, from the year 2000 until June 2018 with a perspective for the short and long term. The results of the long-term tax elasticity were obtained by Ordinary Least Squares and the short-term ones by the Error Correction Model. The estimated parameters indicate that before an increase in GDP, the collection of VAT and ISR grows, also the Tariff Rights against imports.

Keywords: Taxes, elasticities, cointegration.

\section{Introducción}

El estudio presenta una estimación de las elasticidades tributarias de los impuestos: Impuesto al Valor Agregado (IVA), Impuesto sobre la Renta (ISR) frente al Producto Interno Bruto (PIB) y los Derechos Arancelarios (DA) frente a las importaciones a través de modelos econométricos, para lo cual se consideró series de tiempo y de Cointegración.

La investigación interpreta la teoría económica con el manejo eficiente de la política fiscal, encaminada hacia el crecimiento económico, considerando el criterio de diferentes autores y el contexto de país durante el período de estudio, en función de los objetivos económicos del gobierno, los cuales deberían verse acompañados de una reducción del déficit fiscal, incentivos a la inversión y a la apertura de mercados con acuerdos comerciales.

Sobre el tema de elasticidades tributarias existen estudios por Centros de Investigación y Universidades en diferentes países, como en la Universidad de Valencia (España) con el tema de Política Fiscal y Crecimiento Económico, quien señala que uno de los determinantes del bienestar económico y de los niveles de renta per cápita de una economía es la Política Fiscal; en marzo del 2006, en Argentina existe un estudio: "Proyección, acompañamiento y análisis del comportamiento de la recaudación tributaria.", donde se describe que la principal fuente de ingresos públicos en la mayoría de los países es de origen tributario; en Junio del 2008 en México, el Fondo de Cultura Económica, en la publicación de El Trimestre Económico escribieron el tema: "Elasticidad ingreso de los impuestos federales en México: Efectos de la recaudación federal participable", los autores manifiestan que las finanzas públicas de los gobiernos estatales y municipales son muy dependientes de las transferencias federales.

En el año 2008, la Revista Austriana de Economía de las Universidades de Oviedo y Complutense de Madrid publica: "Impuestos y Crecimiento Económico: Una Panorámica": el autor analiza que una medida de política fiscal no afecta la tasa de crecimiento a largo plazo según los modelos neoclásicos de crecimiento, mientras que si desempeña un papel importante en los modelos de crecimiento endógeno; en Octubre del 2011, en Costa Rica el artículo: "Ingresos fiscales y elasticidades tributarias: Estimación de las elasticidades tributarias de corto y largo plazo para los principales impuestos", señala que un gobierno para desarrollar sus tareas necesita contar con suficientes recursos financieros que pueden generarse de distintas fuentes como son los préstamos, venta o explotación de activos de propiedad pública, la emisión de dinero y los tributos e impuestos.

En Octubre del 2016 el Banco Central de Reserva del Perú publicó: "Midiendo el impacto de cambios tributarios sobre la actividad económica en Perú", señala que un aumento de impuestos tiene un efecto negativo y estadísticamente significativo sobre el PIB real; y, en Ecuador en el año 2017, existe un trabajo de titulación titulado: "Elasticidad impositiva en el Ecuador, un estudio para el período 2000 - 2015", los autores manifiestan que en el contexto histórico del Ecuador, el sistema tributario del país ha financiado gran parte del Presupuesto General del Estado, razón por la cual los gobiernos de turno han puesto énfasis en la aplicación de una política fiscal idónea.

\section{Metodología}

El estudio se realizó con datos trimestrales equivalentes a 74 observaciones desde el I trimestre del año 2000 hasta el II trimestre del 2018.

Las series con las que se realizó el análisis econométrico correspondieron a las series trimestrales de recaudación del IVA e Impuesto sobre la Renta (ISR), tomados del Servicio de Rentas Internas (SRI); Ios Derechos Arancelarios (DA) del Banco Central del Ecuador (BCE) - Comercio Exterior. El PIB y las Importaciones del Sistema de Cuentas Nacionales Trimestrales del Ecuador de las tablas de Oferta - Utilización de bienes y servicios. Las series de tiempo, fueron ajustadas a precios constantes del año base $2007=100$ y luego transformadas a logaritmo natural, con el propósito de lograr una dispersión constante.

Para la estimación de las elasticidades de corto y largo plazo, que constituyen el impacto del PIB sobre las recaudaciones tributarias, se siguió una metodología que consta de cinco fases:

Fase I.- Ajuste Estacional de las Series de Tiempo: Las series de tiempo presentan oscilaciones, lo que dificulta observar la tendencia en el tiempo; por ello se aplicó métodos de desestacionalización de variables, con el propósito de obtener resultados precisos y correctos.

Fase II.- Orden de Integración de las Variables y Detección de Cambios Estructurales.- Para determinar el Orden de Integración de las Variables, se analiza la raíz unitaria que presentan las series de tiempo y que permiten una extensión de procesos autorregresivos de orden $\mathbf{p}$ en la hipótesis nula para poder determinar el orden de integración de las mismas. A continuación se prueba Dickey y Fuller Aumentada (ADF) para realizar la contrastación de la hipótesis de significación del parámetro que capta la raíz unitaria o no estacionariedad en la serie.

A continuación, se determina una solución paramétrica a los inconvenientes con los residuos que, aunque son estacionarios, no satisfacen el supuesto de ruido blanco. ADF es un test que incluye una composición de retardos de la variable dependiente que en este caso puede ser el IVA, el ISR y los DA, con el propósito de llegar a obtener el componente autorregresivo para reducir la correlación que otorga el coeficiente de error.

Para la Detección de Quiebres Estructurales, se aplicaron dos pruebas estadísticas, que permitieron determinar la estabilidad de dichas relaciones. En primer lugar se realiza la prueba de CUSUM que calcula la suma de los residuales recursivos conjuntamente con una banda que señala el nivel de confianza a un 95\%, sugiere que si existen residuales fuera de la banda habría una inestabilidad. En segundo lugar se utiliza el método de quiebre de Quandt-Andrews (1960), que es aplicado cuando se desconoce una posible fecha de quiebre estructural y básicamente indica el valor máximo obtenido en varias iteraciones de la prueba de Chow de todas las posibles fechas de cambio o de un eventual rompimiento. Para que el quiebre estructural obtenido sea válido, deberá ser sustentado con posibles acontecimientos económicos que afecten los niveles de recaudación o producción nacional.

Fase III.- Estimación de las Elasticidades en el Largo Plazo.- La estimación se realizó mediante Mínimos Cuadrados Ordinarios, en función del siguiente modelo: $\tau_{t}^{\mathrm{i}}=\beta_{0}+\beta_{1}^{\mathrm{L}} \mathbf{X}_{\mathrm{t}}+\mathrm{AR}(\mathbf{1})+\varepsilon_{\mathrm{t}}$

Donde: $\boldsymbol{\tau}_{\boldsymbol{t}}^{\boldsymbol{i}}=$ Es el logaritmo natural de la recaudación del impuesto al Valor Agregado en el período t; $\boldsymbol{\beta}_{0}=$ Intercepto; $\boldsymbol{\beta}_{\mathbf{1}}^{\boldsymbol{L}}=$ Elasticidad de largo plazo del impuesto correspondiente; $\mathbf{X}_{t}=$ Logaritmo natural del PIB en el período t; $A R(\mathbf{1})=$ Significa que el valor del pronóstico de $X$ en el periodo es simplemente alguna proporción de su valor en el período anterior más un "choque o perturbación aleatoria en el tiempo t".

Para evaluar la estacionariedad de las series se procedió a graficarlas a nive y en primeras diferencias los tributos (ISR y el IVA) con el PIB; y los Derechos Arancelarios (DA) con las Importaciones (M) (todas las series en logaritmos); así mismo se realizó las pruebas de: Dickey - Fuller Aumentada (ADF) y Phillips - Perrón, según esto, se observó que en su forma de nivel la serie no es estacionaria, pero en su forma de primeras diferencias si lo es.

La corrección de autocorrelación se hizo mediante el Proceso Autorregresivo de Primer Orden o AR(1). Cárdenas, et. al (2008) sugiere la introducción de términos autorregresivos en el modelo de largo plazo con el fin de controlar la 
existencia de autocorrelación; dicho proceso se verificó con la prueba de Breusch - Godfrey para especificar el orden de autocorrelación, la cual se probó hasta con cuatro rezagos, según indica la teoría cuando se estudian datos trimestrales.

Fase IV.- Procedimiento de Cointegración de Pesaran, Shin y Smith.- Pesaran, Shin y Smith (2001) proponen una prueba que se conoce como PPS, analiza la existencia de una relación en el largo plazo entre las variables sin la necesidad de conocer previamente el orden de integración de las series.

El estudio de Aguaysa, L., \& Cevallos, D., 2017 encontró lo siguiente:

En la práctica esta prueba se basa en una regresión que relaciona cambios y periodos rezagados en las variables, por lo que se recurre a un modelo autorregresivo de rezagos distribuidos (ARDL). La prueba consiste en demostrar la significancia global de los coeficientes mediante el $\mathrm{F}$ estadístico, aun nivel de significancia del 1\%, 5\% o 10\% (Aguaysa, L., \& Cevallos, D., La elasticidad impositiva en el Ecuador, un estudio para periodo 2000 - 2015, 2017).

Fase V.- Estimación de las Elasticidades en el Corto Plazo mediante el Modelo de Corrección de Errores.- De acuerdo con Cárdenas, O., Ventosa, D., \& Gómez, M., 2008, el modelo de corrección de errores (MCE) permite verificar que las variables están cointegradas y que sus valores en cada período están influenciados por la desviación existente con su equilibrio de largo plazo, lo cual quiere decir que los desequilibrios de corto plazo tienden a corregirse de la siguiente manera: si en el periodo t la recaudación del ISR es relativamente mayor que la elasticidad por la relación de largo plazo con el PIB, entonces, en t+1 la recaudación de ISR debería bajar o el PIB debería subir para retomar el equilibrio de largo plazo.

Para estimar el MCE en primer lugar se obtiene los residuales que otorga el modelo de largo plazo, debido a que las series si cointegran, lo que quiere decir que hay una relación estable de equilibrio en el largo plazo, no obstante en el corto plazo, puede haber desequilibrio. Se modeló en función del siguiente modelo:

$\boldsymbol{\Delta} \boldsymbol{\tau}_{t=\theta_{1}}^{i}\left(\boldsymbol{\tau}_{t-1}^{i}-\beta_{0}+\beta_{1}^{L} Y_{t-1}+\sum_{k=1}^{n i} \cdot \boldsymbol{\beta}_{2 k}^{L}\left(Y_{t-1} \cdot D U_{K t-1}^{T B k} D U\right)+\mu_{1 t} \theta_{1}<0\right.$

$\Delta y_{t}=\theta_{2}\left(\boldsymbol{\tau}_{t-1}^{i}-\beta_{0}+\beta_{1}^{L} Y_{t-1}+\sum_{k=1}^{n i} \cdot \boldsymbol{\beta}_{2 k}^{L}\left(Y_{t-1} \cdot D U_{K t-1}^{T B k} D U\right)+\mu_{2 t} \theta_{2}>0\right.$

Donde: $\mu_{1 t}$ y $\mu_{2 t}$ son ruido blanco, y $\theta_{1}$ y $\theta_{2}$ son parámetros. La ecuación (1) indica la recaudación de impuestos de manera inversa con las desviaciones de largo plazo del período anterior y con los choques estocásticos. La ecuación (2) muestra que el cambio en el PIB se ve afectado directamente por las desviaciones de largo plazo y los choques aleatorios. Al usar el Modelo de Corrección de Errores, implícitamente se está controlando la posible existencia de autocorrelación:

$\Delta \tau_{t}^{i}=\Upsilon 10+\theta_{1} M C E+\sum_{\mathrm{s}=1}^{m \frac{i}{1}} \gamma 11 s \beta \Delta \tau_{t-s}^{i}+\sum_{\mathrm{s}=1}^{m \frac{i}{2}} \Upsilon 12 s \Delta y_{t-s}+\mu_{1 \mathrm{t}}$
$\boldsymbol{\Delta} \boldsymbol{\tau}_{t}^{i}=\Upsilon 20+\theta_{2} M C E+\sum_{s=1}^{m \frac{i}{3}} \Upsilon 21 s \beta \Delta \tau_{t-s}^{i}+\sum_{s=1}^{m \frac{i}{4}} \Upsilon 22 s \Delta y_{t-s}+\mu_{2 \mathrm{t}}$

Donde $\mathrm{m}$ i/j para $\mathrm{j}=1,2,3$ indica el número de rezagos de las diferencias tanto de la variable dependiente como independientes, incluidos para controlar la eventual autocorrelación en los residuales. Los parámetros $\theta_{1}$ y $\theta_{2}$ se interpretan como la velocidad de ajuste, de modo que un valor estimado alto de las $\theta$ implica una mayor respuesta correctiva al desequilibrio observado en el período anterior; también un valor estimado del coeficiente de velocidad de ajuste debe ser estadísticamente diferente de cero, si ambos fueran cero la relación de equilibrio de largo plazo no existiría y el modelo no estaría cointegrado (Cárdenas, O., Ventosa, D., \& Gómez, M., 2008).

\section{Resultados}

\section{Orden de Integración de las Variables y Detección de Cambios Estructurales}

El orden de integración de las series se lo obtiene aplicando la prueba DickeyFuller Aumentada (ADF) y Phillips - Perrón en las series desestacionalizadas y en logaritmos, a nivel y en primeras diferencias.

Tabla 1. Prueba de estacionariedad en todas las series

\begin{tabular}{|c|c|c|c|c|}
\hline \multirow{2}{*}{ Variable } & \multicolumn{2}{|c|}{ Dickey-Fuller Aumentada } & \multicolumn{2}{|c|}{ Phillips-Perrón } \\
\hline & $\mathbf{I}$ & $\mathbf{I}+\mathbf{T}$ & $\mathbf{I}$ & $\mathbf{I}+\mathbf{T}$ \\
\hline Ln_iva & $-1,7183$ & $-2,6668$ & $-1,7922$ & $-2,6125$ \\
\hline Ln_isr & $-3,6691^{*}$ & $-2,7425$ & $-5,5242 *$ & $-7,9009 *$ \\
\hline Ln_darancelarios & $-2,5102$ & $-3,9424 * *$ & $-2,55336$ & $-4,0027^{* *}$ \\
\hline Ln_pib & $-1,6443$ & $-1,039$ & $-1,9064$ & $-1,0298$ \\
\hline Ln_importaciones & $-2,6764 * * *$ & $-1,8297$ & $-3,9948 *$ & $-2,3936$ \\
\hline d_Ln_iva & $-8,8929 *$ & $-8,8917 *$ & $-9,2842 *$ & $-9,4543 *$ \\
\hline d_Ln_isr & $-19,3880^{*}$ & $-20,7296^{*}$ & $-19,4750 *$ & $-20,0220 *$ \\
\hline d_Ln_darancelarios & $-8,5878^{*}$ & $-8,6670 *$ & $-8,6979 *$ & $-8,8471^{*}$ \\
\hline d_Ln_pib & $5,7685 *$ & $-6,0098 *$ & $-5,7116^{*}$ & $-6,0098^{*}$ \\
\hline d__Ln_importaciones & $-4,3899 *$ & $-4,9263 *$ & $-6,7428 *$ & $-9,1632 *$ \\
\hline
\end{tabular}

Los resultados de la prueba ADF y Phillips - Perrón en primeras diferencias analizados (d Ln IVA) en el intercepto (I) y en el intercepto más tendencia $(I+T)$ muestran que se $\mathrm{Rechaza} \mathrm{H}_{0}$ al valor crítico del $1 \%$ y se Acepta $\mathrm{Ha}$, lo que quiere decir que No existe raíz unitaria, la serie es estacionaria en un 99\%. Igual sucede con las otras series, se Rechaza $\mathrm{H}_{0}$ al valor crítico del 1\% y se Acepta $\mathrm{Ha}$, lo que quiere decir que No existe raíz unitaria, las series son estacionarias en un $99 \%$. La identificación de los Cambios Estructurales que fueron identificados mediante las pruebas de CUSUM y Chow consta en las siguientes figuras.

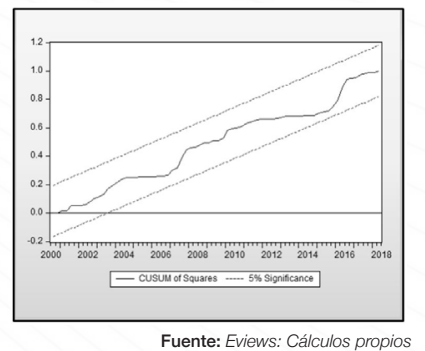

Fuente: Eviews: Cálculos propios
Figura 1. Comportamiento del IVA frente al PIB

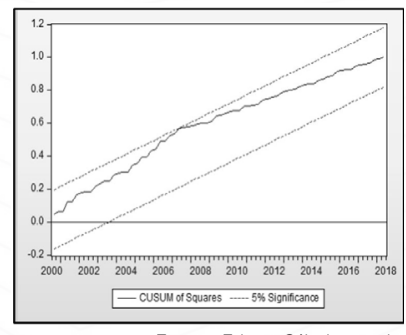

Fuente: Eviews: Cálculos propios Figura 2. Comportamiento del ISR frente al PIB
Las Figuras 1 y 2 muestran que el IVA y el ISR frente al PIB tienen un comportamiento estable, ya que los residuales se encuentran dentro de la banda de confianza.

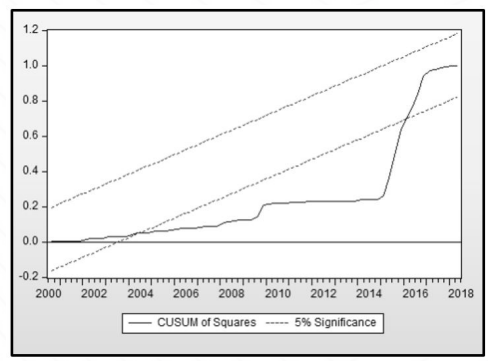

Fuente: Eviews: Cálculos propios

Figura 3. Comportamiento de los Derechos Arancelarios Frente a las Importaciones

Realizando la prueba de CUSUM, según el Figura 3, los Derechos Arancelarios tienen un comportamiento inestable frente a las Importaciones debido a que las medidas de política económica en el ámbito de comercio exterior, no contribuyeron al fomento de las Importaciones, sino mas bien contrajeron la economía ecuatoriana, provocando un desabastecimiento en el mercado interno, producto de ésto, muchas empresas cerraron. La Figura 4 muestra los cambios estructurales.

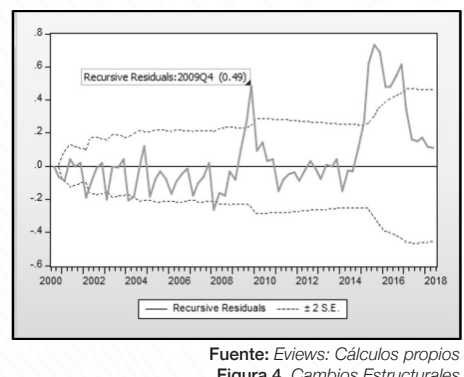

Los cambios estructurales son identificados con la prueba de Chow que permite identificar la fecha más probable de un eventual rompimiento. A continuación la Tabla 2 presenta los cambios estructurales durante el periodo de estudio.

Tabla 2. Detección de Cambios Estructurales

\begin{tabular}{lc}
\hline Período & Prueba de Chow \\
\hline $2009 q 4$ & 10,85 \\
$2015 q 1$ & 81,30 \\
$2015 q 3$ & 50,57 \\
$2016 q 1$ & 18,48 \\
\hline
\end{tabular}


La prueba de Quiebre Estructural de Chow, permite observar que las Importaciones contra los Derechos Arancelarios tuvieron un comportamiento inestable en el cuarto trimestre del año 2009, (2009q4) ocasionado por la imposición de normas técnicas y salvaguardias cambiarias, aplicadas en el Ecuador durante el período 2008 - 2009 a diferentes subpartidas, lo que produjo el cambio estructural en las recaudaciones aduaneras.

El Consejo de Comercio Exterior (COMEX), el 6 de Marzo del 2015 (2015q1), mediante la Resolución 011-2015, aplicó salvaguardias a 2961 subpartidas con tarifas que iban desde el $5 \%$ al $45 \%$ para bienes de consumo, bienes intermedios y de capital con un plazo de 15 meses. Como resultado de éstas medidas, las recaudaciones de aranceles por importaciones se incrementaron en 6,38\% (VELAZQUEZ, 2017).

Para el tercer trimestre del 2015 (2015q3), con la resolución 016-2015 el Comité de Comercio Exterior (COMEX), reforma la resolución 011 - 2015, la cual consistía en incluir a 12 partidas o ítems que serían gravadas con salvaguardias; además, mediante resolución 036-2015 se reforma la resolución anterior, que manifiesta que las sobretasas arancelarias deben ser canceladas en el momento que ingresan al Ecuador vía terrestre o aérea, lo cual incrementa la recaudación.

En el primer trimestre del 2016 (2016q1) las recaudaciones por importaciones disminuyen, debido a la desgravación de las salvaguardias mediante resolución 001-2016 que modificó la sobretasa arancelaria del $45 \%$ al $40 \%$, que afectó a 700 subpartidas. Además se aplica la Resolución 006 - 2016 que elimina el nivel del $5 \%$ de sobretasa arancelaria.

\section{Estimación de la Elasticidad Impositiva a Largo Plazo}

La estimación de las elasticidades se la realiza de mediante Mínimos Cuadrados Ordinarios, introduciendo un AR(1): $T_{t}^{i}=\beta_{0}+\beta^{\llcorner}{ }_{1} X_{t}+A R(1)+\varepsilon_{t}$

Este modelo es adecuado para estimar la relación de largo plazo cuando la variable dependiente (IVA, ISR) esté cointegrada con el PIB; así como los Derechos Arancelarios (DA) con las Importaciones.

Tabla 3. Estimación de las elasticidades de largo plazo

\begin{tabular}{|c|c|c|c|}
\hline Impuesto & $\begin{array}{l}\text { Elasticidad de } \\
\text { Largo Plazo }\end{array}$ & Otros Parámetros Estimados & R-Cuadrado \\
\hline IVA & $\begin{array}{l}\boldsymbol{\tau}_{t}^{i}=1,504074 \\
\text { T-Statistic }=15,58\end{array}$ & $\begin{array}{l}\text { Constante }=-11,14 \\
\text { T-Statistic }=-7,04 \\
\text { Término Autorregresivo AR(1) }=0,728974 \\
\text { T-Statistic }=8,04 \\
\text { Constante }=-25,21\end{array}$ & $\mathbf{R}^{2}=0,98$ \\
\hline ISR & $\begin{array}{l}\boldsymbol{\tau}_{\mathbf{t}}^{\mathrm{i}}=2,324013 \\
\text { T-Statistic }=12,09\end{array}$ & $\begin{array}{l}\text { t-Statistic }=-8,03 \\
\text { Término Autorregresivo AR(1) }=0,036051 \\
\text { T-Statistic }=0,19 \\
\text { Constante }=-2,59\end{array}$ & $\mathbf{R}^{2}=0,68$ \\
\hline DA & $\begin{array}{l}\boldsymbol{\tau}_{\mathbf{t}}^{i}=0,974905 \\
\text { T-Statistic }=6,82\end{array}$ & $\begin{array}{l}\text { T-Statistic }=-1,21 \\
\text { Término Autorregresivo AR(2) }=0,710117 \\
\text { T-Statistic }=8,09\end{array}$ & $\mathbf{R}^{2}=0,85$ \\
\hline
\end{tabular}

Fuente: Eviews: Cálculos propios

Los resultados de las elasticidades estimadas en el largo plazo permiten decir que por cada punto porcentual de aumento en el PIB, la recaudación de largo plazo del IVA se incrementará en 1,50\%, la del ISR en $2,32 \%$ y los DA en $0,97 \%$ cuando las importaciones se incrementan en $1 \%$. Es importante observar que la regresión modelada de los DA frente a las Importaciones presenta un T estadístico no significativo, sin embargo el modelo sirve para la predicción econométrica, por cuanto en su conjunto tiende a ser significativo, ya que $\mathrm{R}^{2}=85 \%$ y el F- Emperico calculado $F=133$

Para verificar la Cointegración se aplicó el método de propuesto por Engle y Granger, que consiste en evaluar la estacionariedad de los residuales del modelo estimado. Seguidamente la tabla:

Tabla 4. Prueba de Cointegración de Engle - Granger

\begin{tabular}{|c|c|c|}
\hline Modelo & Estadístico ADF & Decisión \\
\hline IVA & - $8,205^{* * *}$ & Cointegran \\
\hline ISR & - $\quad 3,584^{* * *}$ & Cointegran \\
\hline $\begin{array}{l}\text { Derechos } \\
\text { Arancelarios }\end{array}$ & $3,015^{* *}$ & Cointegran \\
\hline
\end{tabular}

En la Tabla 4 se detallan los resultados de la prueba ADF a los residuales de cada una las regresiones modeladas. Los resultados de la prueba ADF presentan un comportamiento ruido blanco, es decir que las variables utilizadas están cointegradas de Orden Cero (0) y (I), por ende, las variables tienen una relación de largo plazo, en el caso del IVA y del ISR al 99\% y los Derechos Arancelarios al 95\%.
Estimación de la dinámica en el Corto Plazo a través del Modelo de Corrección Errores (MCE) El MCE permite verificar que las variables están cointegradas, y que sus valores en cada período están influidos por la desviación existente con su equilibrio de largo plazo, lo que quiere decir que si en el período $\mathbf{t}$ la recaudación del IVA es relativamente mayor que la establecida por la relación de largo plazo con el PIB, entonces, en $\mathbf{t + 1}$, la recaudación del IVA debería bajar o el PIB debería subir para retomar el equilibrio de largo plazo. Los resultados constan en la Tabla 5.

Tabla 5. Coeficientes de velocidad de ajuste y correlación Serial de Breusch - Godfrey

\begin{tabular}{llll}
\hline Impuesto & $\begin{array}{l}\text { Variable } \\
\text { Dependiente }\end{array}$ & $\begin{array}{r}\text { Coeficiente de } \\
\text { Ajuste }\left(\theta_{1}, \theta_{2}\right)\end{array}$ & $\begin{array}{l}\text { Breusch } \\
\text { Godfrey }\end{array}$ \\
\hline \multirow{2}{*}{ IVA } & $\Delta$ IVA & $\theta_{1}=-0,243837$ & 2,539064 \\
& $\Delta$ PIB & $\theta_{2}=0,106636$ & 0,134064 \\
ISR & $\Delta$ ISR & $\theta_{1}=-0,943593$ & 0,261033 \\
& $\Delta$ PIB & $\theta_{2}=0,011567$ & 0,138423 \\
DA & $\Delta$ DA & $\theta_{1}=-0,127028$ & 0,271947 \\
& $\Delta \mathrm{M}$ & $\theta_{2}=0,007764$ & 0,275242 \\
\hline
\end{tabular}

Fuente: Eviews: Cálculos propios

Las estimaciones de modelos estimados presentan el signo esperado y se interpretan como la velocidad de ajuste. Un valor estimado alto de las $\theta$ implica una mayor respuesta - correctiva - al desequilibrio observado en el período anterior. Al menos uno de los términos de velocidad de ajuste debe ser estadísticamente diferente de 0 , si ambos fueran 0 , entonces la relación de equilibrio de largo plazo no existiría y el modelo no estaría cointegrado. En este caso podemos observar que el ISR se ajusta más rápido en el siguiente período en un 94\% frente a un ajuste del $24 \%$ del IVA, y los Derechos Arancelarios lo hacen en un 12\%. El coeficiente indica que mientras más cercano a uno sea su valor, más rápido será el ajuste. El número de rezagos fue seleccionado usando el criterio de información Akaike(AIC). También se incluye el estadístico F de la prueba de correlación serial Breusch - Godfrey y con dos rezagos.

Los valores del estadístico F de la prueba de correlación serial Breusch - Godfrey con dos rezagos, indican que no existen problemas de autocorrelación en ninguno de los casos, a excepción del Impuesto sobre la Renta que se ve mejor explicado con un período hacia atrás, coincidiendo con la prueba ADL.

\section{Conclusiones}

En el Ecuador, las reformas tributarias implementadas como medidas de política fiscal por cada régimen gobernante mediante leyes, decretos y resoluciones son continuas; como resultado de éstas, se observa que las recaudaciones tributarias efectivas, según la serie de tiempo analizada (2000 - 2018) del ISR, IVA y DA, no han sido suficientes para el presupuesto general del Estado. En el caso de la Ley Orgánica de Fomento Productivo, Atracción de inversión, Generación de Empleo, Estabilidad y Equilibrio Fiscal, expedida en agosto del 2018, mediante la cual se estableció un régimen de remisiones y reducciones de deudas frente al Fisco, exoneraciones, generación incentivos y beneficios tributarios para la atracción de inversiones privadas, construcción de vivienda de interés social y reglas macro-fis cales y limitación al endeudamiento público no ha dado los resultados esperados.

Los resultados del modelo de elasticidad estimados en el largo plazo indican que por cada punto porcentual de aumento en el PIB, la recaudación de largo plazo del ISR se incrementará en $2,32 \%$, del IVA en 1,50\%, y los DA en 0,97\% cuando las importaciones se incrementan en $1 \%$. De acuerdo a los datos, se tiene que el ISR es la principal fuente de ingresos tributarios para el Fisco, debido a que este impuesto se grava directamente sobre los salarios y sobre la renta de capital, lo cual afecta a la renta de los trabajadores, a las utilidades de los inversionistas, y, a las decisiones relacionadas con el ahorro y la inversión. En el caso del IVA que es un impuesto indirecto gravado a las transferencias de dominio de bienes y servicios realizados por los contribuyentes, también aporta significativamente al PIB. Finalmente, el impuesto a los Derechos Arancelarios con relación a las Importaciones son los tributos que menos contribuyen al crecimiento del PIB, debido a que, éstos dependen del monto de las Importaciones y sus tarifas que son reguladas por la Organización Mundial del Comercio.

Los resultados de la prueba ADF indican que los residuales son estacionarios, presentan un comportamiento ruido blanco, lo cual significa que las variables utilizadas están cointegradas de Orden Cero (0) y (I). Esto quiere decir, que tienen una relación de equilibrio en el largo plazo, en el caso del ISR y del IVA con el PIB en un $99 \%$ y los Derechos Arancelarios en un 95\%. Por lo tanto, se asume que las recaudaciones del ISR y del IVA mantendrán su tendencia en el largo plazo en un 99\%; y, los Derechos Arancelarios con las Importaciones en un 95\%. Esto confirma el requerimiento de una política económica estable, con un sistema tributario confiable, que permita equilibrar las finanzas públicas, cumplir con los compromisos de deuda pública y fomento de la inversión.

En el corto plazo, la estimación de los parámetros de velocidad de ajuste de- 
muestran que si en el período $\mathbf{t}$ la recaudación del IVA es relativamente mayor que la establecida por la relación de largo plazo con el PIB, entonces, en $\mathbf{t + 1}$, la recaudación del IVA debería bajar o el PIB debería subir para retomar el equilibrio de largo plazo. En este caso, el ISR se ajusta más rápido en el siguiente período en un 94\% frente a un ajuste del 24\% del IVA, y los Derechos Arancelarios lo hacen en un 12\%. Esto significa que las recaudaciones del IVA y del ISR en el corto plazo son suceptibles variaciones, ya que dependiendo de la dinamización de la economía y cambios en el sistema tributarrio se tendrá un mayor o un monto menor de recaudación, con una velocidad de ajuste del 94\% en el caso del ISR y del 24\% para el IVA. En el caso del impuesto a los Derechos Arancelarios su nivel de ajuste es bajo (12\%) porque depende del monto de las Importaciones que se realicen en el país.

\section{Referencias}

Aguaysa, L., \& Cevallos, D. (Octubre de 2017). La elasticidad impositiva en el Ecuador, un estudio para periodo 2000 - 2015, 16 - 30.

Cárdenas, O., Ventosa, D., \& Gómez, M. (Junio de 2008). Elasticidad Ingreso de los Impuestos Federales en México. Efectos en la recaudación Federal Participable. Fondo de Cultura Económica, 519 - 531.

Conejo, C., Otoya, M., \& Cardoza, D. (Septiembre de 2011). Ingresos fiscales y elasticidades tributarias. Estimación de las elasticidades tributarias de corto y largo plazo para los principales impuestos, 39-61.

Delgado, F., \& Salinas, J. (2008). Impuestos y Crecimiento Económico: Una Panorámica. Revista Austriana de Economía, 9 - 30.

Doménech, R. (2004). Política Fiscal y Crecimiento Económico. Ekonomi Gerizan, 1-29.

Salim, J. A. (2006). Proyección, acompañamiento y análisis del comportamiento de la recaudación tributaria. 40 Asamblea General del Centro Interamericano de Administraciones Tributarias - Argentina, 1-16.

VELAZQUEZ, V. (Junio de 2017). Efecto de las salvaguardias en la liquidez de las Pymes en el sector comercial del D.M.Q y su impacto en los precios del mercado en el período 2015 al 2016. Trabajo de Titulación. Quito, Ecuador, 81 - 83. 